Ann. Biol. anim., Bioch., Biophys., I965, 5 (I), 53-59.

\title{
EFFETS DE L'INGESTION DE SULFITES SUR L'EXCRÉTION DU CALCIUM CHEZ LE RAT
}

\author{
Denise HUGO'T, J. CAUSERET et J. LECLERC \\ Avec la collaboration technique de W. LOISEL \\ Laboratoire d'Étude des Qualités biologiques des Aliments de l'Homme, \\ Centre national de Recherches zootechniques, Jouy-en-Josas (Seine-et-Oise)
}

\section{SOMMAIRE}

Chez les jeunes rats soumis à un régime alimentaire moyennement riche en calcium $(0,5 \mathrm{p}$. 100 sous forme de carbonate), l'introduction de 0,5 ou I p. 100 de métabisulfite de potassium dans le régime ne modifie pas sensiblement l'excrétion calcique fécale, mais entraîne un accroissement significatif de l'excrétion urinaire.

Inversement, chez des animaux dont le régime est riche en calcium (I p. roo sous forme de carbonate), l'administration de métabisulfite de potassium dans les conditions indiquées précédemment provoque une augmentation significative de l'excrétion calcique fécale, mais reste sans effet sur l'excrétion urinaire.

Ces résultats, dont la signification est discutée, attirent l'attention sur la nécessité de se préoccuper des répercussions éventuelles de l'ingestion des sulfites utilisés en technologie alimentaire comme agents de conservation, sur les modalités de l'excrétion du calcium chez l'Homme.

Parmi les problèmes d'ordre nutritionnel que pose l'emploi des sulfites en technologie alimentaire ( $\left.{ }^{1}\right)$, les effets éventuels de ces agents sur le métabolisme du calcium ne paraissent pas avoir retenu l'attention jusqu'ici.

La question mérite pourtant d'être examinée. En eifet, les sulfites ingérés peuvent être transformés rapidement en sulfates (SOLLMANN, I957 ; I EHMAN, I957), substances qui, dans certaines conditions, accroissent les pertes calciques de l'organisme :

Io Comme l'a montré Kowarski (I958), l'administration orale de sulfate de sodium peut entraîner, chez l'Homme, une augmentation de l'élimination fécale du calcium, augmentation qui serait due à la formation, dans le tube digestif, de sulfate de calcium peu ionisé et difficilement absorbable. Cette propriété a été mise à profit

(1) Au sujet de cet emploi, voir Cluzan, Causeret et Hugot (ig65). 
par l'auteur et par divers autres (Couter, et al., Ig62; LEsTRADET, Ig62) pour lutter contre un passage digestif excessif $d u$ calcium chez certains malades.

Dans les affections qui entraînent une élévation du taux des sulfates sériques (néphropathie chronique, anurie), les sulfates endogènes entraînés par les sécrétions digestives paraissent susceptibles de jouer le rôle du sulfate de sodium administré per os, leur effet se traduisant quelquefois par un bilan calcique négatif (LESTRADET, et al., I 962 ).

$2^{0}$ Dans certains cas, l'ion $\mathrm{SO}_{4}$ accroît l'élimination rénale du calcium. Chez le Rat, l'administration de sulfate de magnésium provoque ce phénomène, alors qu'il ne se produit pas avec d'autres sels magnésiens comme le carbonate, le chlorure ou le citrate (RANDorn et al., I952) ; d'autre part, l'ingestion de sulfate de calcium provoque une perte calcique urinaire beaucoup plus élevée que celle qu'entrainent d'autres sels de calcium (carbonate, lactate, phosphate tricalcique) (CAUsERET et HUGO'T, 1958).

De même, chez l'Homme, l'administration intraveineuse de sulfate de sodium accroît fortement l'élimination du calcium par le rein, cette élimination étant multipliée par 20 et même par 40 dans certaines conditions de perfusion (LESTRADET, Ig62).

Il nous a donc paru utile de chercher si l'ingestion de sulfites ne pourrait pas entraîner des effets similaires.

\section{MATÉRIEL, ET MÉTHODES}

\section{Animaux et régimes alimentaires}

L'expérience a porté sur 49 rats Wistar du sexe mâle, provenant de l'élevage du Laboratoire. Ils étaient âgés initialement de 30 jours et pesaient de 60 à 75 grammes.

Ces animaux ont été répartis en 6 lots qui ont été soumis à des régimes alimentaires dérivant tous du même mélange de base :

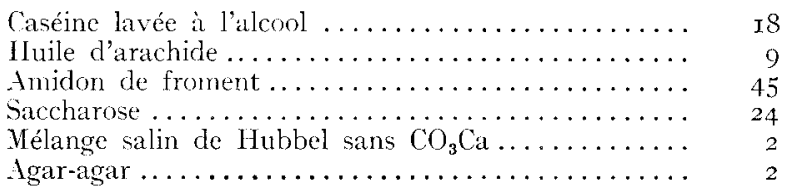

Pour chaque lot, des quantités déterminées de calcium (sous forme de carbonate) et, dans certains cas, de métabisulfite de potassium ( $\left.{ }^{(}\right)$, ont été substituées, dans le mélange précédent, à des quantités d'amidon égales (tabl. I).

La dose de calcium la plus faible $(0,5 \mathrm{p}$. 100 du régime) peut être considérée comme "normale " pour le Rat ; l'autre dose (I p. Ioo) est forte. Le choix de ces deux doses est dû au fait que les conclusions d'études comparatives du genre de celle qui fait l'objet de ce travail dépendent quelquefois du niveau d'ingestion du calcium (CAUSERET' et HugOT. 1959 et 1960).

Le choix des doses de métabisulfite utilisées a été basé sur les données suivantes:

- ces doses peuvent être considérées comme non-toxiques à court terme pour le Rat ;

- les trois valeurs expérimentales du rapport $\frac{\mathrm{SO}_{2} \text { ingéré }}{\text { Ca ingéré }}(0,29$ pour le lot $\mathrm{V} ; 0,58$ pour les lots

(1) Avec le sulfite de sodium, le métabisulfite de potassium est le sulfite le plus employé en France comme agent de conservation. Il foumit une quantité de $\mathrm{SO}_{2}$ égale à $0,58 \mathrm{p}$. 100 de son poids. 
II et VI ; I,I 5 pour le lot III) peuvent être obtenues dans l'alimentation de l'Homme : la quantité de calcium ingéré étant supposée normale (environ $800 \mathrm{mg}$ par jour), ces valeurs correspondraient à une consommation de vin - sulfité à la dose licite maximum de $45^{\circ} \mathrm{mg}$ par litre - égale respectivement à un demi-litre, un litre ou deux litres.

TABLEAU $I$

Teneur des régimes en calcium et en métabisulfite de potassium

\begin{tabular}{|c|c|c|c|}
\hline & & 1 & \\
\hline Lots & $\begin{array}{c}\text { Calcium } \\
\text { (p. } 100 \text { du régrime) }\end{array}$ & $\begin{array}{l}\text { Métabisulfite de } \mathbf{K} \\
\text { (p. } 100 \text { du régime) }\end{array}$ & $\begin{array}{l}\text { Rapport } \\
\mathrm{SO}_{2} / \mathrm{Ca}\end{array}$ \\
\hline I & 0,5 & 0 & 0 \\
\hline II & $0, \overline{3}$ & 0,5 & 0,58 \\
\hline III & 6,5 & 1,0 & 1,15 \\
\hline IV & 1,0 & 0 & 0 \\
\hline V & 1,0 & 0,5 & 0,29 \\
\hline VI & 1,0 & 1,0 & 0,58 \\
\hline
\end{tabular}

Les vitamines ont été introduites dans les six régimes aux doses suivantes, pour $100 \mathrm{~g}$ : thiamine $0,20 \mathrm{mg}$; riboflavine $0,30 \mathrm{mg}$; pyridoxine $0,25 \mathrm{mg}$; acide nicotinique $5 \mathrm{mg}$; pantothénate de calcium I mg; vitamine A $100 \mathrm{UI}$; vitamine $\mathrm{D}_{2} 300 \mathrm{U} \mathrm{I} ; \alpha$-tocophérol I,5 $\mathrm{mg}$; vitamine $\mathrm{K}$ hydrosoluble o,I $\mathrm{mg}$.

Les régimes ont été distribués sous forme de bouillie préparée à partir de 2 parties de mélange sec pour I partie d'eau distillée.

\section{Déroulement de l'expérience}

Après une période d'adaptation de 3 jours, les fèces et les urines des animaux ont été recueillies durant Io jours.

Le dispositif expérimental utilisé a été décrit dans une publication antérieure (CAUSERET, 1954).

\section{Méthode de dosage et calcul des résultats}

Le calcium a été dosé dans les régimes et dans les excreta par complexométrie, au moyen du complexon IV et en utilisant la calcéine comme indicateur (MORI, I959). Le dosage était effectué directement sur les urines. I.es régimes déshydratés, ainsi que les féces déshydratées et pulvérisées, étaient préalablement incinérés à $55^{\circ} \mathrm{C}$ pendant I 2 heures.

Les modalités de l'excrétion du calcium ont été déterminées par calcul des deux rapports :

$$
\frac{\text { Ca fécal }}{\text { Ca ingéré }} \times \text { 100 } \frac{\text { Ca urinaire }}{\text { Ca ingéré }} \times \text { 100 }
$$

\section{RÉSULTATS}

Les résultats obtenus sont réunis dans le tableau 2 .

On voit que, chez les animaux dont le régime renferme $0,5 \mathrm{p}$. Ioo de calcium, l'administration de métabisulfite de potassium ne modifie pas sensiblement l'excrétion fécale du calcium, alors qu'elle entraîne une augmentation significative de l'excrétion urinaire (au seuil de $\mathrm{P}=0.0 \mathrm{I}$ pour le lot II et de $\mathrm{P}=0.05$ pour le lot III). 
TABLEAU 2. - Résultats expérimentaux

\begin{tabular}{|c|c|c|c|c|c|c|}
\hline Lots & $\begin{array}{l}\text { Numéro } \\
\text { des rats }\end{array}$ & $\begin{array}{l}\text { Ca ingéré } \\
\text { (mg/jour) }\end{array}$ & $\begin{array}{l}\text { Ca fécal } \\
\text { (mg/jour) }\end{array}$ & $\begin{array}{c}\text { Ca urinaire } \\
\text { (mg/jour) }\end{array}$ & $\frac{\text { Ca fécal }}{\text { Ca ingéré }} \times 100$ & $\frac{\text { Ca urinaire }}{\text { Ca ingéré }} \times 100$ \\
\hline I & $\begin{array}{c}1 \\
2 \\
3 \\
4 \\
5 \\
6 \\
7 \\
8 \\
\text { Moyennes }\end{array}$ & $\begin{array}{l}55,1 \\
59,0 \\
62,1 \\
55,2 \\
54,6 \\
60,6 \\
55,0 \\
52,8\end{array}$ & $\begin{array}{l}92,3 \\
20,6 \\
29,5 \\
28,9 \\
20,0 \\
22,7 \\
29,2 \\
18,9\end{array}$ & $\begin{array}{l}2,0 \\
1,5 \\
1,75 \\
1,9 \\
1,0 \\
2,0 \\
1,5 \\
1,05\end{array}$ & $\begin{array}{c}41,1 \\
31,9 \\
47,2 \\
52,9 \\
36,6 \\
37,5 \\
42,2 \\
31,6 \\
41,9 \pm 2,3\end{array}$ & $\begin{array}{c}3,6 \\
2,5 \\
2,8 \\
2,3 \\
1,8 \\
3,3 \\
2,7 \\
2,0 \\
2,6 \pm 0,2 \\
\end{array}$ \\
\hline II & $\begin{array}{c}9 \\
10 \\
11 \\
12 \\
13 \\
11 \\
15 \\
\text { Moyennes }\end{array}$ & $\begin{array}{l}48,1 \\
41,2 \\
57,1 \\
47,3 \\
59,7 \\
41,5 \\
55,4\end{array}$ & $\begin{array}{l}21,9 \\
16,1 \\
27,9 \\
27,9 \\
26,1 \\
29,1 \\
29,0 \\
29,0\end{array}$ & $\begin{array}{l}1,35 \\
1,5 \\
2,5 \\
2,5 \\
4,7 \\
1,7 \\
3,7\end{array}$ & $\begin{array}{c}4,5 \\
39,2 \\
48,4 \\
51,3 \\
44,6 \\
52,6 \\
39,7 \\
45,9 \pm 2,0\end{array}$ & $\begin{array}{c}2,8 \\
3,6 \\
4,4 \\
5,3 \\
7,9 \\
4,0 \\
6,7 \\
4,9 \pm 0,7\end{array}$ \\
\hline III & $\begin{array}{c}16 \\
17 \\
15 \\
19 \\
20 \\
21 \\
22 \\
23 \\
24 \\
\text { Moyennes }\end{array}$ & $\begin{array}{l}69,7 \\
55,3 \\
12,9 \\
63,5 \\
58,4 \\
51,9 \\
52,1 \\
52,7 \\
57,1\end{array}$ & $\begin{array}{l}27,3 \\
2: 3,1 \\
16,0 \\
19,9 \\
25,5 \\
19,6 \\
20,1 \\
25,8 \\
25,7\end{array}$ & $\begin{array}{l}2,1 \\
3,15 \\
1,0 \\
2,1 \\
2,6 \\
2,8 \\
2,8 \\
1,5 \\
1,75 \\
1,6\end{array}$ & $\begin{array}{c}42,8 \\
41,7 \\
37, ; \\
30,1 \\
49.5 \\
36,2 \\
38,6 \\
49,0 \\
47,8 \\
40,4 \pm 1,9\end{array}$ & $\begin{array}{r}3,3 \\
6,2 \\
3,3 \\
3,3 \\
4,4 \\
4,4 \\
4,8 \\
2,9 \\
3,3 \\
2,8 \\
3,7 \pm 0,4 \\
\end{array}$ \\
\hline IV & $\begin{array}{c}25 \\
26 \\
26 \\
28 \\
29 \\
30 \\
31 \\
32 \\
\text { Moyennes }\end{array}$ & $\begin{array}{r}117,4 \\
123,6 \\
113,9 \\
108,4 \\
110,8 \\
85,9 \\
101,9 \\
84,4\end{array}$ & $\begin{array}{l}7 i, 7 \\
78,6 \\
67,7 \\
6 i 2,0 \\
70,1 \\
53,8 \\
60,2 \\
100,8\end{array}$ & $\begin{array}{c}9,2 \\
8,0 \\
6,15 \\
12,25 \\
11,0 \\
9,0 \\
10,2 \\
4,3\end{array}$ & $\begin{array}{c}65,3 \\
63,6 \\
59,1 \\
57,-2 \\
63,3 \\
63,1 \\
59,1 \\
72,0 \\
62,8 \pm 1,6\end{array}$ & $\begin{array}{c}7,8 \\
6,5 \\
5,,^{\prime} \\
10,,^{\prime} \\
9,9 \\
10,5 \\
10,0 \\
5,1 \\
8,2 \pm 0,8\end{array}$ \\
\hline V & $\begin{array}{c}33 \\
3 \cdot \mathbf{1} \\
35 \\
36 \\
37 \\
38 \\
39 \\
10 \\
\text { Moyennes }\end{array}$ & $\begin{array}{r}98,3 \\
102,5 \\
99,2 \\
94,0 \\
102,4 \\
93,4 \\
77,5 \\
89,2\end{array}$ & $\begin{array}{l}68,7 \\
73,7 \\
77,4 \\
75,7 \\
71,1 \\
73,1 \\
49,1 \\
60,6\end{array}$ & $\begin{array}{l}7,1 \\
9,8 \\
9,3 \\
6,1 \\
9,1 \\
6,1 \\
6,7 \\
5,8\end{array}$ & $\begin{array}{c}69,9 \\
71,9 \\
78,0 \\
80,5 \\
69,7 \\
78,4 \\
63,3 \\
73,7 \\
73,2 \pm 2,0\end{array}$ & $\begin{array}{c}7,5 \\
9,6 \\
9,4 \\
6,5 \\
8,9 \\
6,6 \\
8,7 \\
7,0 \\
8,0 \pm 0,4\end{array}$ \\
\hline VI & $\begin{array}{c}41 \\
42 \\
43 \\
4, k \\
45 \\
46 \\
47 \\
48 \\
49 \\
\text { Moyennes }\end{array}$ & $\begin{array}{r}101,8 \\
105,8 \\
81,2 \\
80,7 \\
64,1 \\
97,5 \\
81,7 \\
109,6 \\
102,8\end{array}$ & $\begin{array}{l}70,6 \\
81,5 \\
6-2,3 \\
51,9 \\
11,9 \\
71,4 \\
31,4 \\
73,6 \\
61,9\end{array}$ & $\begin{array}{l}7,7 \\
6,: 2 \\
9,3 \\
7,7 \\
6,0 \\
5,9 \\
4,5 \\
3,0 \\
3,6\end{array}$ & $\begin{array}{c}69,4 \\
79,9 \\
73,9 \\
68,0 \\
65,4 \\
73,2 \\
66,6 \\
67,2 \\
63,1 \\
09,6 \pm 1,7\end{array}$ & $\begin{array}{c}7,6 \\
5,9 \\
11,0 \\
9,6 \\
9,4 \\
6.0 \\
5,5 \\
2,7 \\
3,5 \\
6,8 \pm 1,1\end{array}$ \\
\hline
\end{tabular}


Inversement chez les animaux dont le régime renferme I p. Ioo de calcium, le sulfite accrôit significativement l'excrétion fécale du calcium (au seuil de $P=0.0$ I pour le lot $\mathrm{V}$ et de $\mathrm{P}=0.02$ pour le lot $\mathrm{VI}$ ), mais il reste sans effet sur l'excrétion urinaire.

Enfin, quel que soit l'apport alimentaire de calcium, l'excrétion calcique - fécale et urinaire - n'est pas modifiée significativement lorsque la teneur du régime en métasulfite de potassium passe de $0,5 \mathrm{p}$. I00 à I p. IOO.

\section{DISCUSSION}

Avant de tenter d'expliquer la nature du lien observé entre la valeur de l'apport calcique et les effets de l'ingestion de sulfite sur les modalités de l'excrétion du calcium, il n'est pas inutile de rappeler que nous avons déjà signalé des liens du même genre. Ainsi :

- lorsque le taux de calcium du régime est peu élevé, la substitution de carbonate de calcium au lait dans ce régime ne modifie pas l'excrétion calcique du Rat; mais lorsque 1'apport calcique est élevé, cette même substitution accroît l'excrétion fécale du calcium et diminue 1'excrétion urinaire (CAUSERET et HUGoT, I960) ;

- lorsque le taux de calcium du régime est peu élevé, la substitution d'oxalate de calcium au carbonate dans ce régime accroit l'excrétion fécale du calcium sans modifier l'excrétion urinaire; mais lorsque l'apport calcique est élevé, cette môme substitution augmente à la fois l'excrétion fécale et l'excrétion urinaire (CAUSERET et Hugot, I96I).

Dans le cas présent, tout se passe comme si le sulfite n'entravait l'absorption digestive du calcium que dans la mesure oì l'apport calcique est excédentaire. Cette constatation s'accorde avec le fait que, chez le Rat en croissance, soumis à un régime de taux calcique "normal ", l'ion $\mathrm{SO}_{4}$ n'accroît pas l'élimination fécale du calcium (RANDOIN et al., I952 ; CAUSERET et Hugot, I958). Rappelons aussi que le tube digestif du Rat absorbe assez activement le calcium d'un sel peu ionisé et réputé difficilement absorbable comme l'oxalate, pourvu que le régime soit peu riche en calcium ; si l'apport calcique est élevé, la fraction du calcium alimentaire non absorbée augmente (CAUSERET et Hugot, I96I).

Il est plus difficile d'expliquer que l'accroissement de l'excrétion urinaire du calcium sous l'influence de sulfite ne se manifeste qu'avec le régime à $0,5 \mathrm{p}$. Ioo de calcium. Puisque, chez le Rat, le rein élimine difficilement le calcium, il est possible qu'un " plafond " de l'excrétion soit déjà atteint chez les sujets du lot IV, l'introduction de sulfite dans les régimes des lots V et VI ne pouvant donc plus l'augmenter encore.

L'existence d'un tel plafond conduit d'ailleurs à se demander si l'accroissement de 1'excrétion de calcium fécale chez les animaux des lots V et VI est bien due à une diminution de l'absorption digestive. Il se pourrait en effet que l'influence des sulfites sur le métabolisme du calcium s'exerce au seul niveau de la rétention, les sujets des lots $\mathrm{V}$ et VI rejetant dans l'intestin l'excès de calcium absorbé, parce que le rein 
ne peut plus l'éliminer. Il serait intéressant d'étudier la valeur de cette hypothèse en effectuant des bilans calciques avec dilution isotopique $\left({ }^{45} \mathrm{Ca}\right)$.

Enfin, on peut s'étonner que les effets du métabisulfite de potassium sur l'excrétion calcique ne s'accroissent pas lorsque le taux de ce corps dans le régime passe de 0,5 p. Ioo (lots II et V) à I p. Ioo (lots III et VI). Toutefois, s'il est possible que les sulfites agissent sur l'excrétion du calcium par l'intermédiaire des sulfates auxquels ils donnent naissance in rivo, on ne doit pas perdre de vue que la possibilité qu'a l'organisme d'oxyder les sulfites est limitée : il n'est donc pas à exclure que la capacité d'oxydation maximum soit atteinte chez les animaux des lots II et $\mathrm{V}$ dont le régime est déjà relativement riche en sulfites.

Quoi qu'il en soit, il serait injustifié de tirer de notre travail des conclusions applicables avec certitude à l'alimentation humaine. On sait en effet que les modalités du transit du calcium dans l'organisme ne sont pas les mêmes chez le Rat et chez 1'Homme. En particulier, chez ce dernier, 1'absorption digestive du calcium paraît moins complète et l'excrétion du calcium par le rein représente une fraction de l'excrétion totale beaucoup plus élevée. Nous nous bornerons donc à conclure que les effets des sulfites observés chez le Rat rendent nécessaire une expérimentation sur 1'Homme lui-même.

Reçu pour publication en noiembre 1964 .

\title{
SUMMARY
}

\author{
EFFECI OF INGESTION OF SULPHITES ON EXCRETION OF CALCIUM IBY RATS
}

Ingested sulphites can be rapidly changed to sulphates within the organism. Many workershave shown, in man and rat, that when sulphates are given then can increase the amount of cal cium excreted in faces and urine. The authors therefore studied whether sulphites, substances widely used as preservatives in food technology, had a similar effect on the elimination of calcium.

Forty-nine young rats received balanced diets with 0,5 or 1.0 p. Ioo calcium, as carbonate, and 0.5 or 1.0 p. 100 potassium metalbisulphite was added to some diets. Excreta were collected during Io days.

In the rats given the diet with $0.5 \mathrm{p}$. Ioo calcium, $0.5 \mathrm{p} .100$ potassium metabisulphite did not affect excretion of calcium in fæces but it caused a significant increasc of the excretion in urine.

Inversely, in the animals given 1.0 p. 100 calcium in the diet, metabisulphite given in the conditions already described calused a significant increase in excretion of calcium in fæces but had no effect on the excretion in urine.

'The significance of the results is discussed. 'They draw attention to the need for study of the eventual effect that ingestion of sulphites may have on excretion of calcium by man.

\section{RÉFÉRENCES BIBLIOGRAPHIQUES}

Causkret J., I954. Recherches sur l'utilisation physiologique du calcium au cours de la croissance chez le Rat. Nouveaux ispects du rôle biologique de la vitamine D. Ann. Zootech., 3, 27.

Causeret J., Hugot D., 1958. Influence du taux calcique du régime alimentaire sur l'utilisation physior logique du calcium d 2 divers sels. I. Ftude comparative du carbonite, du sulfate et du lactate de calcium. Ann. Zoolech., 7, 60.

Causeret J., Hugot D., I959. L'oxalate de calciun constitue-t-il une source de calcium utilisable poule Rat en croissance? $C$. R. Acad. $S c i ., 249$, r $2 \gamma^{2} 2$. 
Causeret J., liugot D., ig6o. Iifficacité calcique comparée d'une poudre de lait et du carbonate de calcium, en fonction de la teneur en calcium du régime alimentaire. C. R. Acal. Sci., 1960, 250, 401.

Causeret J., IItcot D., ig6r. L'efficacité biologique de l'oxalate de calcium chez le jeune Rat. Effet de la vitamine $\mathrm{D}_{2}$. Bull. Soc. Chim. biol., 43, $47 \mathrm{I}$.

Cluzhin R., Catseret J., Hugot D., i 965 . lítude de toxicité à long teme sur le métabjulfite de potassium (chez le Rat). Ann. Biol. anim. Bioch. Biophys., 5 (sous presse).

Coutel Y., Jezequel C., Borxel J., Noury S., rg62. Trois cas d'hypercalcémie chez l'enfant, traités par le sulfate de soude. Arch. tr. Pediat., 19, 1033.

Kowarski A., 1958. Idiopathic hypercalcemia : treatment with sodium sulfate. Pedialrics, $22,533$.

Lehmany A. J., 1957. Association of Food and Drug Officials of the Cinited States, 21, 29.

Lestradeí H., ig62. Diététique et calcium. Journée annuelle de Nitrilion el de Diététique (Hólel-Dieu, Paris), P. 57 .

Lestrade's II., Frederici A., Rodriguez-Soriano J., igfo. L'hypersulfatemie n'est-elle pas la cause des ostéodystrophies de l'insuffisance rénale chronique? Presse míl., 70, 2 I.

MoRI K., 1959. Direct microcomplexometric analysis of calcium in biological materials. Arch. Biochem Biophys., 83, 552 .

Randon L., Causeret J., Il tgot D., Morel G., i952. Influence de divers sels magnésiens administrés par voie orale sur la rétention du calcium dans l'organisne. Bull. Soc. Chim. biol., 34, II59.

Soldmax T., I957. 1 . Hamual of Phamacology, ge édit., W. B. Saunders Co, p. I53. 